\title{
Les libertins érudits ou la morale des corrompus
}

\author{
Laurence Tricoche-Rauline
}

\section{(2) OpenEdition}

Journals

Édition électronique

URL : http://journals.openedition.org/edl/921

DOI : $10.4000 /$ edl. 921

ISSN : 2296-5084

\section{Éditeur}

Université de Lausanne

\section{Édition imprimée}

Date de publication : 15 décembre 2015

ISBN : 978-2-940331-47-5

ISSN : 0014-2026

\section{Référence électronique}

Laurence Tricoche-Rauline, "Les libertins érudits ou la morale des corrompus », Études de lettres [En ligne], 3-4 | 2015, mis en ligne le 01 décembre 2018, consulté le 15 décembre 2020. URL : http:// journals.openedition.org/edl/921 ; DOI : https://doi.org/10.4000/edl.921 


\section{LES LIBERTINS ÉRUDITS OU LA MORALE DES CORROMPUS}

Les libertins sont regardés par les dévots comme des malades, susceptibles de contaminer le corps social avec leurs raisonnements pervertis. Pour leurs adversaires, leur liberté de pensée n'a aucune valeur philosophique. Elle sert seulement de justification à leur goût pour la débauche. Sous le masque, par prudence face aux pouvoirs, ils défendent en effet l'importance des désirs, y compris sexuels. Mais sont-ils pour autant corrompus? Ils n'hésitent pas à se réapproprier la notion de corruption, pour accuser les jésuites d'immoralité. Ils savent prendre la posture du moraliste. Pour eux, seule la recherche du juste milieu garantit la liberté et la tranquillité du sage. Leur morale n'est toutefois pas celle de la faute, qui fait de tout homme un pécheur. Le libertinage tente de réconcilier l'homme avec sa nature, qui est corruptible, soumise à la maladie et aux ravages du temps. Ils invitent ainsi à considérer la corruption, libérée de tout discours moralisateur, comme indissociable de l'existence, à laquelle elle vient même donner une partie de sa valeur.

Suite à la Réforme, les catholiques doivent tirer les conséquences de la fin de l'unité de l'Eglise. Les polémiques religieuses s'en trouvent avivées. Laccusation de corruption devient l'un des instruments privilégiés de condamnation d'un parti par un autre. Elle contribue à légitimer, pour les protestants, le schisme par rapport à l'Eglise catholique et romaine, accusée de promouvoir le vice. Elle est reprise par les catholiques contre leurs adversaires protestants et libertins, éloignés de l'orthodoxie ${ }^{1}$. Sur le plan politique et social, les guerres de religion sont la traduction concrète

I. Procédant par amalgame, l'orthodoxie catholique condamne aussi bien les protestants que les libertins, qui se seraient affranchis du respect dû à l'Eglise et à ses pratiques pour obéir aux exigences de leur orgueil et de leur esprit critique. 
et tragique de ces dissensions, également favorisées par l'état de relative faiblesse du pouvoir, suite à l'assassinat d'Henri IV, qui était parvenu en 1598 grâce à l'Edit de Nantes à mettre en sourdine les tensions religieuses les plus violentes ${ }^{2}$. S'ouvre à ce moment une période de transition, considérée par certains prédicateurs comme plus propice à un certain relâchement des mœurs et de la piété.

De ce fait, certains s'inquiètent d'une contagion de la débauche et de l'athéisme ${ }^{3}$, qui pourrait entraîner une corruption du corps social, nettement plus inquiétante encore que celle des individus. Mersenne, par exemple, dans L'Impiété des déistes, athées et libertins de ce temps (1624) avance le chiffre de 50’000 athées vivant à Paris en 1623, ce dont il fait un signe de la corruption des temps. Les libertins, c'est-à-dire ceux qui sont suspects de s' être "affranchis ${ }^{4}$ " des dogmes philosophiques, moraux et religieux, sont désignés comme corrompus et comme potentiellement corrupteurs pour la jeunesse, car capables de séduire des esprits soucieux de plaisirs et de liberté, que l'on retrouverait en particulier au sein de la noblesse et à la cour. Pour Garasse, dans La Doctrine curieuse des beaux esprits de ce temps ou prétendus tels (1623), de même que pour Mersenne, le libertinage n'est pas une position philosophique claire et cohérente, mais plutôt une posture orgueilleuse destinée à donner une légitimité au relâchement moral et à la débauche. Face à ces accusations très graves, certains libertins se défendent, en dénonçant la corruption de leurs

2. L'Edit de Nantes est toutefois loin d'avoir résolu toutes les difficultés auxquelles le christianisme se trouve confronté. Subsistent des tensions importantes entre catholiques et protestants, qui peuvent se sentir floués par l'Edit, mais aussi entre gallicans et ultramontains. Les questions relatives à l'application du concile de Trente et au statut des jésuites ne sont pas non plus résolues. Les dissensions se retrouvent au plus haut niveau de l'Etat. Jusqu'en 1630, moment où Louis XIII la contraint à l'exil, le roi, sous l'influence de Richelieu, se montre favorable à une alliance avec les puissances protestantes, alors que Marie de Médicis défend une alliance avec les puissances catholiques.

3. Les progrès supposés de l'athéisme peuvent être expliqués par l'influence en France du libertinisme italien, ainsi que par le renouveau de la pensée sceptique. Voir T. Gregory, Genèse de la raison classique de Charron à Descartes, ainsi que R. Popkin, The History of Scepticism.

4. Etymologiquement, le terme «libertin» renvoie à libertinus, qui désigne l'affranchi, c'est-à-dire un homme libéré de son statut d'esclave, mais que l'on ne saurait considérer comme un citoyen à part entière. Pour une réflexion sur la notion de libertinage, voir J.-P. Cavaillé, "Libertinage, irréligion, incroyance, athéisme dans l'Europe de la première modernité (XVI'-XVII ${ }^{\mathrm{e}}$ siècle)». 
accusateurs, souvent jésuites ${ }^{5}$. Ils montrent que la fausse dévotion, qui se pare d'arguments réputés incontestables pour dissimuler des intentions plus politiques que morales, et parfois inavouables, est le seul véritable facteur de corruption pour l'ordre social. La morale est pour chacun des partis le point de référence au nom duquel la critique de l'adversaire est menée. Dans les deux cas, derrière l'accusation de corruption se trouve celle de dissimulation et d'hypocrisie, qui conduirait les uns et les autres à se donner toutes les apparences de la vertu. Les jésuites dénoncent la culture du secret et les stratégies prudentielles des libertins, qui choisissent parfois de publier sous couvert d'anonymat ou en usant de différents pseudonymes. Ils en font un signe condamnable d'élitisme ${ }^{6}$. Mais sous la pression des pouvoirs politiques et religieux, les libertins ne peuvent se présenter explicitement comme critiques à l'égard des dogmes, vérités philosophiques et religieuses réputées incontestables. En réponse aux attaques dont ils sont victimes, ils dénoncent la dissimulation par les jésuites de leur immoralité et de leurs ambitions, sous le masque de la foi. Malgré la violence des conflits qui les opposent, libertins et dévots ont, paradoxalement, un système de valeurs commun: ils partagent un idéal de sincérité, le refus de l'abandon au vice et la crainte d'une mise en danger du corps social par la dissolution des repères moraux et religieux.

La corruption est donc une notion qui sert d'instrument à la polémique entre les libertins, supposés corrompus, mais qui adoptent volontiers la posture du moraliste, et les apologètes, qui se posent en défenseurs de la religion chrétienne. Si les libertins remettent en cause les certitudes morales et religieuses, c'est uniquement pour une élite "déniaisée", capable d'éviter toute confusion entre liberté, plaisir et irresponsabilité. Leur sagesse n'est pas l'immoralité. Mais ils se réapproprient également

5. Les libertins reprennent certains éléments du discours anti-jésuite - l'accusation de corruption, le soupçon d'opposition au pouvoir - tel qu'il s'est développé en France, en particulier après l'assassinat d'Henri IV. Théophile, pour sa défense, dénonce l'acharnement des pères Voisin et Garasse, qui seraient à l'origine d'une véritable campagne de propagande contre lui. Les jésuites constitueraient selon lui un contre-pouvoir dangereux et d'autant plus inquiétant qu'il est omniprésent dans le monde.

6. Voir F. Garasse, La Doctrine curieuse des beaux esprits de ce temps ou prétendus tels, p. 1. Le jésuite prête aux libertins cette maxime, censée justifier le choix du secret et qu'il s'attache à réfuter: "Il y a fort peu de bons esprits au monde, et les sots, c'està-dire, le commun des hommes, ne sont pas capables de notre doctrine: et partant il n'en faut pas parler librement, mais en secret et parmi les bons esprits, confidans et cabalistes". 
la notion de "corruption", d'une manière discrètement hétérodoxe, pour la dépouiller de son sens essentiellement moral et théologique ${ }^{7}$. Ils ne considèrent pas que la nature humaine soit déchue dès l'origine. Si l'on refuse toute forme de culpabilisation, il apparaît que la corruption est surtout le signe de notre inscription dans la temporalité et qu'elle est un mouvement indissociable, au sein de la nature, de celui de la création. Elle est l'une des conditions du renouvellement du monde ${ }^{8}$. La religion, qui diffuse le faux espoir du salut et de l'immortalité, inviterait à résister à cette nécessité, et interdirait ainsi aux hommes de trouver leur juste place dans les limites étroites que leur condition pourtant leur impose.

\section{La «corruption» des libertins}

De même que le terme «libertinage», qui renvoie étymologiquement à l'idée d' "affranchissement", la notion de "corruption" est indissociable de celle d'une rupture de l'individu par rapport au monde et au groupe. Le «corrompu» est celui qui a "rompu» avec ses anciens modes d'appartenance. Le discours chrétien fait de la "corruption» des libertins, à la fois cause et conséquence de leur autonomie à l'égard des contraintes morales et religieuses, un élément à charge essentiel, dans le procès qu'il leur intente. Garasse, dans La Doctrine curieuse des beaux esprits de ce temps ou prétendus tels, leur reproche d'être gourmands, ivrognes, impudiques et abandonnés à une vulgaire recherche du plaisir. Leur prétention à l'intelligence ne s'accorderait pas avec les incohérences de leur pensée. Garasse ne prend pas au sérieux leurs positions religieuses, et en particulier leur athéisme, dont il fait un simple prétexte leur permettant de justifier leur immoralité. Pour lui, les libertins sont l'un des symptômes les plus inquiétants d'une maladie du corps social, vicié par une immoralité face à laquelle la répression serait le seul véritable remède 9 .

7. Ce terme renvoie au concept grec de phtora, que la philosophie traduit par le terme de "corruption", et qu'elle oppose au terme genesis, qui désigne la génération, l'épanouissement, la naissance.

8. Les libertins n'ignorent pas l'atomisme et les réflexions de Démocrite, Epicure et Lucrèce. Lorsque les atomes se séparent, ils ne retournent pas au néant. Leur séparation est le préalable à une nouvelle union, qui est à l'origine de la génération.

9. Garasse considère, par exemple que: "quand un esprit se met et abandonne à mal faire, c'est une gangrène irrémédiable, il faut couper, trancher, brûler de bonne heure, 
Le procès et la condamnation de Théophile de Viau, qui meurt des suites de sa captivité en 1626, marquent une évolution très nette dans l'histoire du libertinage, dont l'expression se fait ensuite plus dissimulée et moins "flamboyante ${ }^{10}$ » à mesure que s'affirme le pouvoir absolu de Louis XIV.

Pour les défenseurs de la religion chrétienne, au fondement de la «corruption» libertine se trouve le désir de se présenter comme un individu autonome, qui pourrait légitimement exercer son esprit critique face à la Loi. Les libertins, par l'importance qu'ils accordent au désir ainsi qu'aux passions, signalent leur distance à l'égard des discours moraux et religieux qui les condamnent. Ils redonnent à la sexualité toute sa place, que la Chute lui avait retirée. Ils jugent avec sévérité le spectacle du monde, qui leur inspire le rire ou une certaine mélancolie, expressions parfois très proches de la supériorité du sage, qui trouvent leur origine dans le constat de l'incertitude et de l'instabilité universelles ${ }^{11}$. Ils choisissent également très souvent d'écrire à la première personne, pour rendre compte de leurs propres expériences et pour s'affirmer en tant qu'individus face à un monde qui ne les comprend pas, voire qui les opprime ${ }^{12}$. L'homme que les libertins donnent à voir, à travers leurs écrits personnels, est incarné: il ne saurait être simplement considéré comme un "animal raisonnable», tel que le définit Aristote. Il est certes un esprit, mais surtout un corps, inscrit dans le temps et dans l'espace, un être

autrement l'affaire est désespérée» (p. 16).

Io. Cette expression de «libertinage flamboyant» est utilisée par René Pintard, qui a joué un rôle majeur dans l'historiographie du libertinage (voir sa célèbre thèse: Le libertinage érudit dans la première moitié du XVII siècle, dont la première publication date de 1943), pour qualifier un mode d'expression relativement libre des libertins, toléré par le pouvoir entre 1610 et 1623 - date de l'emprisonnement de Théophile de Viau -, en particulier dans l'entourage de Gaston d'Orléans, et dont seule la défaite de la Fronde marque la fin définitive. L'impiété doit ensuite s'inscrire dans les étroites limites du for intérieur, et les libertins envisager de complexes stratégies d'écriture et de dissimulation.

II. Le livre de l'Ecclésiaste, qui proclame la "vanité» de toute chose, est une référence dont on retrouve souvent la trace dans les textes libertins et qui peut être à l'origine d'une certaine mélancolie du sage.

I2. Dans la première moitié du siècle, c'est le cas de Théophile, dans la Première Journée (1623), ainsi que de Tristan L'Hermite, dans Le Page disgracié (1643). Pour les libertins, le choix de l'écriture personnelle a certes un sens esthétique, mais il traduit également le souci de s'affirmer librement face aux exigences de la morale, qui condamnent le discours sur soi - on connaît par exemple la réprobation que Pascal fait peser sur l'écriture de Montaigne, dans Les Essais - et d'affirmer une forme d'individualisme. Voir L. Tricoche-Rauline, Identité(s) libertine(s). 
dont l'imagination et les désirs ne sauraient être négligés et qui résiste à tout discours culpabilisant.

Cette importance accordée aux désirs, y compris sexuels, conduit les défenseurs de la religion chrétienne à accuser les libertins d'obscénité. Cette accusation se fonde sur la parution à cette époque de divers recueils licencieux ${ }^{13}$. Mais l'obscénité, qui n'est pas toujours dénuée d'intentions blasphématoires, est un objet de dissimulation pour les libertins, qui n'assument que sous le masque de pseudonymes ou de l'anonymat les conséquences que de telles publications pourraient avoir pour eux. Jean-Christophe Abramovici affirme que «la corruption offre [aux yeux du moraliste] un défaut ou un excès de visibilité; qu'il dénonce la multiplicité de ses masques ou celle de ses formes, l'obscénité est un leitmotiv de son discours ${ }^{14}{ }^{\prime}$. A une époque où s'impose un strict contrôle du corps, sous l'influence des exigences morales et religieuses, l'obscénité est dénoncée comme une transgression fondamentale des règles de la vie en société. Les libertins défendent au contraire une vision déculpabilisée de la sexualité, définie comme un élan vital, naturel et comme un facteur essentiel de plaisir. Théophile, par exemple, convoque de nombreux motifs liés au désir et à son assouvissement. Dans le sonnet "Je songeais que Phyllis des enfers revenue", il énonce même cette conclusion, qui semble autoriser la femme à une sacrilège profanation de l'âme, placée quasiment sur le même plan que le corps au sein de la sexualité: "Comme tu t'es vanté d'avoir ...tu mon corps,/ Tu te pourras vanter d'avoir ...tu mon âme ${ }^{15}$ ». On peut également citer l'exemple des Confessions de Jean-Jacques Bouchard (1606-1641), œuvre dans laquelle la sexualité, évoquée sous l'angle de l'impuissance et de la crainte qu'elle lui inspire, est radicalement étrangère à toute considération morale. Pour les libertins, l'idéalisme et les contraintes que la morale et la religion imposent en matière amoureuse sont des illusions inefficaces, qui n'empêchent pas l'individu de répondre à la puissance irrépressible de ses désirs, et qui ont de surcroît pour conséquence de lui infliger le poids insupportable de la culpabilité.

13. Les recueils suivants sont révélateurs de cette "obscénité» libertine: le Cabinet satyrique, ou recueil des vers piquans et gaillards de ce temps (1618), Les Délices de la poésie française (1620), La Quint-essence satyrique (1622), ou encore le célèbre Parnasse satyrique (1623), qui faillit coûter le bûcher à Théophile.

I4. J.-Ch. Abramovici, Obscénité et classicisme, p. 42.

I5. Th. de Viau, Euvres poétiques. Sonnet, p. 130 
L'accusation la plus grave dont les libertins sont victimes en matière de morale, car elle peut mener sur le bûcher et qu'elle est souvent confondue avec celle d'hérésie ${ }^{16}$, est sans aucun doute celle de «sodomie». Garasse a ces mots très durs pour évoquer ce suprême péché de chair, preuve supplémentaire et irréfutable de l'immoralité libertine, qui conduit à adopter des pratiques réputées contre-nature:

Jadis lors qu'il y avoit encores un peu de sentiment et de pieté dans les ames des bons vieux françois, au seul nom de sodomie, on ne parloit que de brusler tout vif celuy qui en eus esté seulement soupçonné, et aujourd'huy on verra un livre qui se vend publiquement dans les galeries du palais, qui porte en front un sonnet execrable, par lequel l'autheur, qui se dit le Sieur Theophile se repentant, à ce qu'il dit, d'avoir eu et contracté une maladie infame avec une prostituée, fait vœu à Dieu d'estre sodomite tout le reste de ces jours, et ce par des parolles les plus execrables qui soient jamais sorties de la bouche du plus abominable sodomite qui ait esté enveloppé dans les cendres de Gomorrhe. Helas! Flammes de Sodome, ou estes vous! Puis que les hommes ferment les yeux! Que ne fondés vous sur cette abomination! Que ne vengés vous les querelles de Dieu vostre maistre, duquel on profane le nom, que ne consumés vous en cendres ces livres plus impudiques que ne furent jamais les maisons et les murailles de Seboim, d'Adama, de Sodome, et de Gomorrhe? ${ }^{17}$

Le jésuite craint pour Paris le même sort que certaines des villes antiques ${ }^{18}$. Il fait allusion à un sonnet de Théophile intitulé "Phyllis, tout est ...tu, je meurs de la vérole», et qui comporte cette audacieuse conclusion:

Mon Dieu, je me repens d'avoir si mal vécu:/ Et si votre courroux à ce coup ne me tue,/ Je fais vœu désormais de ne ...tre qu'en cul ${ }^{19}$.

I6. Dassoucy, dans ses Aventures, désigne l'homosexualité comme une "hérésie en fait d'amour" (Aventures burlesques de Dassoucy, p. 133.) L'accusation de sodomie a par exemple conduit Chausson et Paulmier à avoir la langue coupée et à être brûlés vifs pour viols et enlèvement d'enfants en 1661 .

I7. F. Garasse, Doctrine, p. 782 sq.

I8. Sodome, Gomorrhe, Adama et Seboïm sont des villes pécheresses et maudites, que Dieu, dans sa colère, a détruites. De ces villes de la vallée de Siddim, seule la cinquième, Zoar, est épargnée.

19. Th. de Viau, Euvres poétiques, p. 358. 
Théophile reprend ainsi le vœu chrétien de chasteté, pour le détourner et en faire un vœu libertin de sodomie. Pour ses accusateurs, il incarne ainsi de manière paroxystique la débauche et la corruption, par son choix supposé d'une sexualité orientée exclusivement vers le plaisir et rompant radicalement avec l'impératif de la procréation, qui est pourtant, pour les dévots, son seul mode possible de légitimation.

La prudence à laquelle les libertins sont contraints rend, pour les défenseurs de la religion chrétienne, leurs comportements forcément suspects et objets d'un certain nombre de spéculations, relevant parfois en partie du fantasme. L'expression du libertinage est considérée comme à la fois excessivement spectaculaire - on craint qu'elle n'influence la noblesse - et dissimulée, ce que les apologètes interprètent comme un signe de la lâcheté et de l'hypocrisie des libertins ${ }^{20}$. A la dénonciation de la corruption sur le fond s'ajoute celle d'une forme corrompue, marquée par le choix de la dissimulation, en partie imposé aux libertins par les circonstances historiques et politiques. Les apologètes dénoncent l'usage du double langage, des ressources de la fiction et la pratique de l'ironie, qui conduit à déresponsabiliser le langage et qui rend difficile à définir l'espace véritable de la pensée. Pour les libertins, qui défendent néanmoins toujours un idéal de sincérité et de transparence absolue, les mots, dans une société corrompue et marquée par le poids du mensonge, ne peuvent plus renvoyer avec certitude aux choses. Les libertins critiquent non seulement la Vérité, mais ils mettent également en question la possibilité même d'accéder à une vérité grâce à la raison, qu'elle soit philosophique, morale ou religieuse ${ }^{21}$. Leurs adversaires considèrent comme intolérable

20. Pour les libertins, la crainte de la répression culmine après le procès de Théophile et la prise en main du pouvoir par Richelieu en 1624. Elle conduit par exemple Charles Sorel à réécrire son Histoire comique de Francion. Entre la version de 1623 et celle de 1626, beaucoup des allusions libertines ont été supprimées. La nouvelle version qu'il présente de son œuvre en 1633 est même attribuée à un autre auteur: Moulinet Du Parc, dont le récit s'inspirerait du témoignage de Francion lui-même. La paternité de l'œuvre libertine est fictionnelle, en partie incertaine.

2I. Certains libertins - c'est le cas de La Mothe Le Vayer, par exemple - face aux vérités religieuses, qu'ils considèrent comme relatives et incertaines, défendent la suspension sceptique du jugement. On peut considérer qu'il s'agit de protéger la foi des attaques éventuelles du rationalisme. Mais la suspension du jugement fait de l'adhésion aux dogmes religieux le fruit d'un certain aveuglement, d'une volonté de croire, parfaitement contingente. Le fidéisme des libertins peut être interprété comme le masque de leur athéisme. 
cette corruption du signe linguistique, forme abusivement dissociée de ses liens essentiels avec l'être. Jean-Christophe Abramovici relève même dans l'œuvre de Molière cette dissociation entre la forme irréprochable et le sens profond des pièces, moralement audacieux. Il considère que «l'obscénité (fondamentale, idéologique) de Molière, c'est d'avoir effacé de ses pièces les obscénités (formelles, choquantes, mais insignifiantes) de l'ancienne comédie pour mieux en perpétuer la morale corrompue. ${ }^{22}$ " La politesse, le souci de ne pas choquer, passe même paradoxalement, aux yeux des dévots, pour le signe inquiétant d'une plus grande corruption de l'être. Pour les libertins, elle signale une adaptation au monde nécessaire à leur survie, mais dont ils font un signe de la corruption du monde lui-même.

\section{Les libertins moralistes: la critique de la corruption du monde}

Les libertins répondent aux critiques de leurs adversaires, qui créent une image polémique et fictionnelle du libertin, en leur renvoyant des accusations comparables. Les jésuites sont accusés d'impiété, d'immoralité et d'hypocrisie. Ils seraient eux-mêmes corrompus, et de plus cautionneraient la corruption des hommes du monde. Guy Patin, considérant qu'ils trahissent l'idéal de Jésus, note:

Paris abonde en gens qui haïssent ces Pères à force de les bien connaître: qui ont l'impudence de s'appeler Compagnons de Jésus, combien que le bon Seigneur n'ait jamais appelé personne son compagnon, que Judas même, qui le vendit ${ }^{23}$.

22. J.-Ch. Abramovici, Obscénité et classicisme, p. 44. Dans la «Préface» du Tartuffe, Molière évoque l'accusation de corruption sur le fond, aussi bien que sur la forme, dont sa pièce est l'objet. Il mentionne ainsi les réactions des spectateurs à la représentation de sa comédie: «les uns l'ont considérée dans sa pureté, lorsque les autres l'ont regardée dans sa corruption, et confondue avec tous ces vilains spectacles qu'on a eu raison de nommer des spectacles de turpitude" (Molière, Euvres complètes, tome II, p. 886).

23. G. Patin, Lettres. Lettre à Charles Spon, p. 434. Patin, qui défend les libertés gallicanes, est très hostile aux jésuites, ultramontains et favorables au pouvoir $\mathrm{du}$ Pape. Il critique leurs mœurs sexuelles dissolues, et en particulier leur propension à la sodomie, leur cupidité, leur ambition. Il a également une certaine admiration pour les jansénistes et accueille avec satisfaction la publication des Provinciales (1656-1657) de Pascal, œuvre majeure de la polémique anti-jésuite. 
Théophile organise également sa défense, dans son procès, en insistant sur les vices de ses accusateurs ${ }^{24}$. C'est une parole pamphlétaire qu'il développe contre Garasse. Le jésuite est pour lui avant tout soucieux de gloire et de pouvoir, et il exerce sur la société l'influence pernicieuse qu'il prête à son adversaire libertin. Théophile précise qu'au moment de son procès, " $[\mathrm{s}]$ es accusateurs faisaient retentir les églises de médisances, dont l'Hôtel de Bourgogne eût été scandalisé. ${ }^{25}$ " Ce procès est l'occasion de dissiper l'illusion de la moralité des représentants de l'Eglise. La religion est le masque idéal du vice, dont seuls les plus crédules sont les dupes.

En moralistes, les libertins dénoncent plus généralement les vices de leurs contemporains, et en particulier l'orgueil. Dans Le Page disgracié, Tristan L'Hermite montre qu'il a conscience des critiques auxquelles le choix de l'écriture personnelle expose un auteur. C'est pourquoi il affirme que, dans son œuvre, "la Vérité [se] présentera seulement si mal habillée qu'on pourra dire qu'elle est toute nue. On ne verra point une peinture qui soit flattée. ${ }^{26}$ " Les libertins mettent également en cause le poids de l'argent et le désir de pouvoir, qui ont pour conséquences l'ascension sociale des plus médiocres et la persécution des sages, forcément incompris. Ils critiquent la corruption du présent et font volontiers l'éloge des temps passés. Théophile déplore en particulier la solitude du sage face à l'universelle corruption intellectuelle et morale de la société. Il fait ainsi cet amer constat:

Aujourd'hui l'injustice a vaincu la raison,

Les bonnes qualités ne sont plus de saison,

La vertu n'eut jamais un siècle plus barbare,

24. Cyrano de Bergerac fait également une satire très violente d'un jésuite, dans ses Lettres satiriques et amoureuses, p. 133, ainsi que dans Autres lettres. Contre un Jé[suite] assassin et médisant, p. 213. Le personnage qu'il décrit, Messire Jean, est caractérisé par sa violence, son hypocrisie et son immoralité. Cyrano fait même allusion à la légitimation par les jésuites du tyrannicide. Il fait au «jésuite assassin et médisant» cette remarque ironique: «Assurément vous me preniez pour un roi, quand vous prêchiez vos disciples de m'assassiner, mais ce n'est pas de toute farine que se font les Châtels, et les Ravaillac» (p. 213).

25. Th. de Viau, Apologie au Roi, in Euvres complètes, tome III, p. 259.

26. T. L'Hermite, Le Page disgracié, in Euvres complètes, tome I, p. 207. 
Et jamais le bon sens ne se trouva si rare ${ }^{27}$.

Du fait de la généralisation du mensonge et de la dissimulation, en particulier à la cour, il considère qu'il est devenu difficile de distinguer le vice et la vertu. Les exigences de la morale ne voudraient plus rien dire, pour une partie de ses contemporains. La cour, lieu de pouvoir censé donner l'exemple du raffinement et de la politesse mondaine, se révèle marquée par la violence, voire par la barbarie des courtisans, ces "esclaves insensés des pompes de la Cour ${ }^{28}$ ». Garasse, dans son acharnement contre Théophile, ne serait finalement que le porte-parole d'une foule agressive et stupide, toujours perçue comme une menace pour l'intelligence et pour la vérité. Dans l'avertissement à L'Histoire comique de Francion, la "corruption" des temps devient la justification légitime de la dissimulation, d'une écriture qui voile son véritable sens, "pource que l'on n'aime pas aujourd'hui à voir la vérité toute nue ${ }^{29} \%$. Le héros, Francion, est même placé à la tête d'une confrérie des «Braves et des Généreux", qui se fixe pour objectif de lutter contre la corruption des mœurs ${ }^{30}$. Cette posture de moraliste serait censée rendre vaine le discours de ceux qui dénoncent le libertinage. Dans son œuvre, Sorel montre que "c'est bien perdre son temps de vouloir critiquer celui qui est le critique des autres ${ }^{31}$ ». En proposant d'eux, dans leurs écrits personnels, des autoportraits en sages dénonçant les vices d'autrui, les libertins entendent corriger l'image d'incarnations de la corruption, laissée par la créature décrite et fustigée par Garasse.

27. Th. de Viau, Elégie à une dame, in Euvres complètes, p. 102. Théophile, dans la Satire seconde, pose également cette question: "Où se cache aujourd'hui la vertu de jadis?» (p. 132).

28. Ibid., p. 248.

29. F. Sorel, Histoire comique de Francion, p. 384.

30. C'est ainsi qu'il décrit leurs activités: "Nous n'attaquions pas seulement le vice à coups de langue: le plus souvent, nous mettions nos épées en usage et chargions sans merci ceux qui nous avaient offensés» (ibid., p. 286). Francion pointe la discordance très fréquente entre le pouvoir conféré aux gens par l'argent et le véritable mérite, intellectuel et moral. Il se montre toutefois sans illusion sur ses chances de réussir dans cette mission, étant donné l'universalité de la corruption: «Il me semblait que, comme Hercule, je ne fusse né que pour chasser les monstres de la terre; toutefois, pour dire la vérité, il n'y avait pas moyen que j'opérasse du tout en cela, car il faudrait détruire tous les hommes qui n'ont plus rien maintenant d'humain que la figure» (ibid., p. 298).

3I. Ibid., p. 385. 
Les libertins entendent prouver que le libertinage ne se confond pas avec le vice, mais qu'il suppose au contraire la modération et la maîtrise des passions, autant de qualités auxquelles la société contemporaine serait étrangère. Ils goûtent les plaisirs de la bonne chère, mais veillent à ce que le plaisir reste dans les limites de la raison et ne sombre pas dans l'excès. La sagesse du mangeur est un juste milieu entre les vaines contraintes de la politesse mondaine et les excès prétentieux des faux savants, qui révèlent leur véritable nature dans la débauche. Dans la Première journée, Théophile défend un rapport apaisé avec la nourriture qui devrait être acceptée comme un besoin naturel du corps, lié aux plaisirs et aux joies de la conversation. Les libertins refusent également l'ivrognerie, et se révèlent même relativement méfiants à l'égard du vin, susceptible d'entraîner l'ivresse et la perte de maîtrise de soi. Sorel, dans L'Histoire comique de Francion, prête à son héros ces très sages propos:

C'est à faire aux esprits bas [...] à ne pouvoir de telle sorte commander sur eux-mêmes qu'ils ne sachent restreindre leurs appétits et leurs envies. Pour moi, bien que j'aime ce breuvage autant qu'il est possible, je m'abstiendrai facilement d'en goûter et je ferais ainsi de toute autre chose que je chérirais uniquement ${ }^{32}$.

Le rapport des libertins à la sexualité ne se confond pas non plus avec la licence. Les poèmes licencieux ont une fonction libératoire. Ils constituent essentiellement une provocation par rapport à un ordre moral très contraignant. Il importe toutefois de ne pas tirer de conclusions, à partir de ces poèmes, sur les comportements effectifs. Les libertins défendent les plaisirs en matière de sexualité, mais en aucun cas l'asservissement à une autre forme de tyrannie, tout aussi insoutenable que celle des pouvoirs politiques et religieux: celle du désir.

La valorisation de la liberté est pour eux indissociable du sens des responsabilités. La liberté ne s'identifie ni au désordre intérieur des passions, ni à l'anarchie, sur le plan social. Les libertins, même s'ils ne sont pas des défenseurs acharnés de la monarchie absolue et de l'ordre politique, moral et religieux qu'elle impose, n'entendent pas prendre le risque du désordre. En réservant la lecture de leurs œuvres à une élite "déniaisée», capable de lire "entre les lignes ${ }^{33}$ ", ils montrent qu'ils ne

32. Ibid., p. 70.

33. Voir L. Strauss, La persécution et l'art d'écrire. 
sont ni corrompus, ni corrupteurs pour la société: seuls les lecteurs intelligents sont susceptibles de comprendre une pensée originale et audacieuse, que le "vulgaire» confondrait avec une défense de l'immoralité.

\section{La reprise détournée et libertine de la notion de corruption}

Les libertins pratiquent fréquemment, en matière morale et religieuse surtout, «le détournement dissimulé du discours de l'adversaire ${ }^{34}$ ». C'est ainsi qu'ils privent la notion de corruption de son sens moral et religieux. Le terme "corruption", pour les chrétiens, renvoie à la nature de l'homme, marquée par le péché originel, qui l'a précipité dans la souffrance et dans la temporalité. Pour espérer gagner le salut, l'homme ne devrait oublier ni sa nature corrompue ni l'espoir qu'incarne pour lui le Christ ${ }^{35}$. Les libertins s'efforcent au contraire d'effacer le poids de la Chute sur l'histoire des hommes. Cyrano évoque même le scandale que constitue cette généralisation à l'humanité entière d'une responsabilité pourtant individuelle:

Hélas! pour un seul fruit qu'Adam mangea, cent mille personnes moururent qui n'étaient pas encore; l'arbre même est forcé par la Nature de commencer le supplice de ses enfants criminels ${ }^{36}$.

L'homme ne peut être considéré comme coupable d'une faute qu'il n'a pas commise.

Les libertins, parfois inspirés par une vision matérialiste du monde ${ }^{37}$, conçoivent la corruption comme l'altération du corps, soumis aux

34. Voir S. Gouverneur, Prudence et subversion libertines.

35. Voir B. Pascal, Pensées: «Et tout ce qu'il nous importe de connaître est que nous sommes misérables, corrompus, séparés de Dieu, mais rachetés par Jésus-Christ» (p. 76 sq.). La double nature de l'homme fait qu'il n'est pas simplement corrompu, il a aussi le souvenir de la perfection qui le caractérisait avant la Chute, et qu'il aspire à retrouver. Les libertins sont ceux qui oublient la corruption originelle de l'homme, ce qui les précipite dans l'orgueil.

36. Cyrano de Bergerac, Lettres. Contre l'automne, p. 56. Pour Cyrano, la pluralité des mondes, thèse qu'il défend dans Les Etats et Empires de la Lune et du Soleil, contredirait d'ailleurs l'universalité de la Chute.

37. Cyrano de Bergerac, Lettres amoureuses, p. 189: "L'homme a deux trépas à souffrir sur la terre, l'un violent, qui est l'amour, et l'autre naturel, qui nous rejoint 
ravages du temps ou à la maladie ${ }^{38}$. Tristan L'Hermite, dans le poème $A$ des cimetières, propose un tableau centré sur la vanité des choses de ce monde. Il décrit les "ossements entassés» et les tombes: «Représentant la vie et sa fragilité,/ Pour censurer l'orgueil des âmes insolentes ${ }^{39}$. Les libertins évoquent l'inéluctable affaiblissement, voire la décomposition naturelle du corps après la mort. Cette forme de corruption que nous ne maîtrisons pas, même si les textes peuvent suggérer des moyens audacieux de l'éviter ${ }^{40}$, n'occasionnera pour nous aucune souffrance. Les libertins usent des lieux communs du sommeil ou de la nuit pour évoquer le silence et l'obscurité de la mort. Celle-ci ne serait donc rien, elle serait le simple terme de l'existence et seul le moment du passage de vie à trépas pourrait éventuellement être craint ${ }^{41}$. Des Barreaux, qui se réapproprie sur le mode libertin, laïcisé, le motif du memento mori, incite également ses lecteurs à une certaine sérénité face à la mort:

à l'indolence de la matière." Dans Les Etats et Empires de la Lune et du Soleil, le héros se trouve confronté à cette vision de la mort étrangère à toute forme d'idéalisation et centrée sur la corruption du corps: il lui est donné à imaginer un "cadavre marchant sur les vers dont il regorge, à la merci des crapauds qui lui mâchent les joues» (p. 138).

38. M. de Montaigne, dans cet extrait (III, 12), fait le lien entre corruption et maladie: "En ces maladies populaires, on peut distinguer sur le commencement, les sains des malades: mais quand elles viennent à durer, comme la nostre, tout le corps s'en sent, et la teste et les talons: aucune partie n'est exempte de corruption. Car il n'est air, qui se hume si gouluement: qui s'espande et penetre, comme faict la licence». La corruption serait en partie liée à l'état de société. Elle est nettement moins présente à l'état de nature, chez ceux que l'Europe considère comme des sauvages. Montaigne évoque la force physique et morale des "cannibales", qui sont moins soumis aux maladies que les européens et qui restent étrangers à bien des vices: «En ceux-là [les sauvages] sont vives et vigoureuses les vrayes et plus utiles et naturelles vertus et proprietez, lesquelles nous avons abstardies en ceux-cy, et les avons seulement accommodées au plaisir de nostre goust corrompu" (Les Essais, I, 31, p. 205).

39. T. L'Hermite, Les Amours. A des cimetières, in Euvres complètes, tome II, p. 53.

40. Cyrano de Bergerac, dans Les Etats et Empires de la Lune (p. 139-140), décrit une scène d'anthropophagie, qui est présentée comme un moyen d'éviter l'ensevelissement du corps et de faire participer la mort au cycle naturel de la vie. La corruption, par la digestion, dans le corps des proches du défunt, devient une condition nécessaire à la régénération de la matière. Dans cette vision cyclique du temps, la corruption a toute sa place, et ne s'oppose plus à la genèse, dont elle est même l'un des préalables.

4I. Th. de Viau, A Philis. Stances, in Euvres complètes, p. 73. «Le Soleil meurt pour moi, une nuit m'environne,/ Je pense que tout dort,/ Je ne vois rien, je ne parle à personne:/ N'est-ce pas être mort?». La mort est d'autant moins inquiétante qu'elle n'est pas entièrement étrangère à la vie. La mélancolie, la souffrance amoureuse peuvent donner une image de ce qu'elle est, pour celui qui est pourtant bien vivant. 
Mortel, qui que tu sois, n'aye plus à frémir/ De l'horreur de la mort et de la sépulture,/ Ce n'est qu'un doux repos où tombe la Nature,/ Dont l'insensible estat ne doit faire gémir ${ }^{42}$.

La corruption est le signe de notre mortalité, qui fait partie intégrante de la vie et qui devrait nous inciter à nous tourner vers le plaisir, au présent.

Les libertins ne reconnaissent donc pas la nature de l'homme comme corrompue au sens moral, bien au contraire. Ils rappellent que c'est le plus souvent l'état de société qui a conduit à la corruption, du fait des discours moraux et religieux, qui éloignent l'homme de son aspiration naturelle à la vie. En imposant d'étroites limites au désir, qui n'aurait la possibilité de s'exprimer de manière légitime que dans le cadre restreint du mariage, la société détourne les individus des exigences de la création, auxquelles elle préfère la préoccupation mortifère de la corruption, qu'il importerait de sans cesse racheter ${ }^{43}$. Dans la Satire première, Théophile de Viau évoque les conséquences néfastes des discours d'autrui sur l'âme naturellement bonne de l'individu:

Je crois que les destins ne font venir personne

En l'être des mortels qui n'ait l'âme assez bonne,

Mais on la vient corrompre, et le céleste feu

Qui luit à la raison ne nous dure que peu;

Car l'imitation rompt notre bonne trame,

Et toujours chez autrui fait demeurer notre âme ${ }^{44}$.

Ce sont les discours moralisateurs qui sont aliénants pour l'homme. Ils figent son esprit dans des postures qui ne sont pas celles qu'il avait adoptées à l'origine. En matière de morale, il s'agirait d'opérer une forme

42. A. Adam, Les libertins au XVII' siècle, p. 198.

43. Voir Cyrano de Bergerac, Les Etats et Empires de la Lune et du soleil, p. 143: «que les Grands de votre monde sont enragés de faire parade d'un instrument qui désigne un bourreau, qui n'est forgé que pour nous détruire, enfin l'ennemi juré de tout ce qui vit; et de cacher, au contraire, un membre sans qui nous serions au rang de ce qui n'est pas, le Prométhée de chaque animal, et le réparateur infatigable des faiblesses de la nature». Cyrano évoque ainsi le paradoxe d'une société qui valorise l'épée, signe de noblesse, mais aussi instrument de destruction, au détriment du «membre viril», qui est pourtant à l'origine de la génération. Ce paradoxe est le signe d'une société que les discours moraux et religieux ont affaiblie, qui considère la vie comme une menace et qui se révèle obsédée par la culpabilité et par la mort.

44. Th. de Viau, Satire première, in Euvres complètes, p. 130 sq. 
de révolution copernicienne. Pour Théophile, ce n'est pas la nature de l'homme qui doit changer, car elle est au cœur de son existence et lui apporte le «céleste feu» nécessaire à sa raison. C'est l'homme qui doit accepter de s'adapter à sa nature et de soumettre sa vie sociale aux exigences qu'elle lui fixe, pour éviter à son âme de "demeurer" dans les discours d'autrui, signe d'un immobilisme trompeur et stérile. Dans son Histoire comique, Sorel semble rêver d'un temps où «les lois naturelles seraient alors révérées toutes seules et [où] l'on vivrait comme au siècle d'or. ${ }^{45}$ " Ce rêve est celui d'un retour à l'innocence et au bonheur que l'homme pourrait retrouver en reconquérant non pas le jardin d'Eden ${ }^{46}$, mais son propre jardin, sa nature, que les discours moraux et religieux l'ont abusivement obligé à renier.

L'argument de la corruption est donc au cœur de la polémique entre les libertins et les apologètes, qui l'utilisent chacun de leur côté pour dénoncer le parti adverse, au nom d'une défense commune des valeurs morales. Pour les libertins, le respect de la morale ne peut toutefois être le fruit d'une culpabilisation outrancière des individus, fondée sur le mythe du péché originel et de la Faute. Le discours religieux précipiterait au contraire les individus dans le mensonge et dans la transgression. Il sert essentiellement de justification à l'asservissement de la majorité, au profit exclusif de quelques-uns, détenteurs de tous les pouvoirs. Pour les libertins, la posture du moraliste est l'occasion d'une remise en question de la conception théologique de l'homme. L'histoire ne conduit, avec le triomphe du christianisme, qu'à la culpabilisation et au raidissement dans le dogmatisme et l'hypocrisie. La morale des libertins "corrompus" invite à une rupture à l'égard des discours moralisateurs et de la haine de soi qu'ils professent. En acceptant un rapport apaisé avec sa nature, à laquelle il est d'ailleurs illusoire de croire que l'on peut imposer silence, et en retrouvant une forme de souveraineté qu'il avait perdue, l'homme doit pouvoir accéder à l'autonomie et au plaisir. Ce plaisir ne naît pas de l'abandon au vice. Il est la réponse bien tempérée, dans les limites

45. Ch. Sorel, Histoire comique de Francion, p. 401.

46. Cyrano de Bergerac imagine à de multiples reprises un tel retour de l'homme au Paradis, en particulier dans Les Etats et Empires de la Lune et du Soleil. Ce retour se solde par un échec et n'apporte pas au personnage le salut espéré. Il le précipite au contraire dans une suite de mésaventures dont certaines auraient pu lui coûter la vie. 
imposées par le souci de préserver la sérénité de l'individu, aux désirs humains les plus légitimes.

Pour les libertins, la corruption qui frappe l'homme ne peut être l'objet d'une quelconque condamnation morale. Elle est une conséquence physique inévitable de la maladie et de la mort. Elle est plus symboliquement le fruit des incessants changements qui traversent nos existences marquées par la temporalité, et dont l'individu peut faire une chance, une occasion de rompre avec le poids des permanences et des liens d'appartenance qui pèsent sur lui, au lieu de les subir comme un signe de malédiction. Contrairement aux dévots, qui nous imposent de faire de la recherche du Salut la fin véritable de l'existence, les libertins nous invitent à nous tourner vers la vie et vers l'espoir d'un bonheur terrestre fondé sur la satisfaction des désirs et étranger à la débauche. Ils font de la corruption le seul fondement possible d'une morale respectueuse de l'individu et la condition d'une nouvelle genèse, qui accepterait enfin le désir et le corps comme parties intégrantes de notre humanité, ainsi réconciliée avec elle-même.

Laurence TRICOCHE-RAULINE Université de Saint-Etienne 


\section{BIBLIOGRAPHIE}

\section{Textes}

Cyrano de Bergerac, Savinien de, Lettres satiriques et amoureuses, éd. par Jean-Charles Darmon, Paris, Desjonquères, 1999.

—, Les Etats et Empires de la Lune et du Soleil, éd. par Madeleine Alcover, Paris, Honoré Champion, 2004.

Dassoucy, Charles Coypeau, Aventures burlesques de Dassoucy, préface par Emile Colombey, Paris, Adolphe Delahays, 1858.

Garasse, François, La Doctrine curieuse des beaux esprits de ce temps ou prétendus tels, Paris, Chappelet, 1624.

Molière, Euvres complètes, Paris, Gallimard, Bibliothèque de la Pléiade, 1972, tome II.

Montaigne, Michel de, Les Essais, éd. par Pierre Villey, Paris, PUF, 1999.

Pascal, Blaise, Pensées, éd. Philippe Sellier, Paris, Presses Pocket, «Agora», Les Classiques, 2003.

Patin, Guy, Lettres. Lettre à Charles Spon, in Libertins du XVII e siècle, éd. par Jacques Prévot, Paris, Gallimard, Bibliothèque de la Pléiade, 2004, p. XXX.

Sorel, Charles, Histoire comique de Francion (éd. de 1633), éd. par Fausta Garavini, Paris, Gallimard, Folio, 1996.

Tristan L'Hermite, Le Page disgracié, in Euvres complètes, éd. par Jean Serroy, Paris, Honoré Champion, 1999.

VIau, Théophile de, Euvres poétiques, Sonnet, éd. Guido Saba, Paris, Classiques Garnier, 1990.

—, Euvres complètes, éd. par Guido Saba, Paris, Honoré Champion, 1999, tome III. 


\section{Travaux}

Abramovici, Jean-Christophe, Obscénité et classicisme, Paris, PUF, 2003.

Adam, Antoine, Les libertins au XVII siècle, Paris, Buchet/Chastel, 1964.

Cavaillé, Jean-Pierre, «Libertinage, irréligion, incroyance, athéisme dans l'Europe de la première modernité (XVIe-XVII e siècle)", Les dossiers du Grihl, 2007-02 (http://dossiersgrihl.revues.org/642).

Gouverneur, Sophie, Prudence et subversion libertines. La critique de la raison d'Etat chez François de La Mothe Le Vayer, Gabriel Naudé et Samuel Sorbière, Paris, Honoré Champion, 2005.

Gregory, Tullio, Genèse de la raison classique de Charron à Descartes, Paris, PUF, 2000.

Popkin, Richard, The History of Scepticism: from Savoranola to Bayle, New York/Oxford, Oxford University Press, 2003.

Strauss, Léo, La persécution et l'art d'écrire, Paris, Presses Pocket, "Agora», 1989.

Tricoche-Rauline, Laurence, Identité(s) libertine(s): l'écriture personnelle ou la création de soi, Paris, Honoré Champion, 2009. 
